\title{
Prolonged Drug-Drug Interaction between Terbinafine and Perphenazine
}

\author{
Young-Min Park $\bowtie$ \\ Department of Psychiatry, Ilsan Paik Hospital, Inje University College of Medicine, Goyang, Republic of Korea
}

I report here an elderly woman receiving perphenazine together with terbinafine. After 1 week of terbinafine treatment she experienced extrapyramidal symptoms and, in particular, akathisia. Her symptoms did not disappear for 6 weeks, and so at 2 weeks prior to this most recent admission she had stopped taking terbinafine. However, these symptoms persisted for 3 weeks after discontinuing terbinafine. It is well known that terbinafine inhibits CYP2D6 and that perphenazine is metabolized mainly by CYP2D6. Thus, when terbinafine and perphenazine are coadministrated, the subsequent increase in the concentration of perphenazine may induce extrapyramidal symptoms. Thus, terbinafine therapy may be associated with the induction and persistence of extrapyramidal symptoms, including akathisia. This case report emphasizes the importance of monitoring drug-drug interactions in patients undergoing terbinafine and perphenazine therapy.

Psychiatry Investig 2012;9:422-424

Key Words Terbinafine, Perphenazine, Drug-drug interaction, Akathisia, Extrapyramidal symptoms.

\section{INTRODUCTION}

It is well known that terbinafine inhibits CYP2D6 and that perphenazine is metabolized mainly by CYP2D6. ${ }^{1}$ Thus, when terbinafine and perphenazine are coadministrated, the subsequent increase in the concentration of perphenazine may prove fatal. For example, extrapyramidal effects, such as dysarthria, dystonia, tardive dyskinesia, and parkinsonism are common adverse effects of perphenazine. In addition, a neuroleptic malignant syndrome, consisting of fever, rigidity, tachycardia, and cardiac arrhythmias, has also been reported from the use of perphenazine. ${ }^{2}$ However, more importantly, terbinafine remains in the body for a long time after discontinuation of the drug. ${ }^{3}$ Surprisingly, some researchers have found therapeutic concentrations of terbinafine in finger and toe nails for as long as 36 weeks after discontinuation of the drug. ${ }^{4}$ Thus, even after the discontinuation of terbinafine, prolonged pharmacokinetic drug interactions between terbinafine and

Received: June 24, 2012 Revised: August 8, 2012

Accepted: August 9, 2012 Available online: November 12, 2012

$\triangle$ Correspondence: Young-Min Park, MD, PhD

Department of Psychiatry, Ilsan Paik Hospital, Inje University College of Medicine, 170 Juhwa-ro, Ilsanseo-gu, Goyang 411-706, Republic of Korea

Tel: +82-31-910-7260, Fax: +82-31-910-7268, E-mail: medipark@hanmail.net

(a) This is an Open Access article distributed under the terms of the Creative Commons Attribution Non-Commercial License (http://creativecommons.org/licenses/bync/3.0) which permits unrestricted non-commercial use, distribution, and reproduction in any medium, provided the original work is properly cited. perphenazine can occur and the adverse effect of perphenazine can persist without current terbinafine medication.

I present here the case of an elderly woman receiving perphenazine and terbinafine. Several days after the commencement of this combined medication, she experienced extrapyramidal symptoms and, in particular, akathisia. However, her symptoms persisted for 3 weeks after discontinuing terbinafine. This is the first case report of a prolonged drug-drug interaction between perphenazine and terbinafine.

\section{CASE}

An elderly female was admitted to my hospital with severe akathisia, generalized weakness, anxiety, insomnia, gait disturbances, bradykinesia, and depressed mood. Her main concern was the severe akathisia. The finding for laboratory analysis, electrocardiogram, urinalysis, and brain MRI were all within the respective normal ranges at pretreatment. She had experienced a depressive episode 15 years previously, since when she had received psychiatric medication at a local psychiatric clinic until admission to our hospital. Although she had not experienced any further depressive episodes, her insomnia persisted, and so she had continued to take her drugs. For several years she had been taking the following medications:, lorazepam (1 mg), diazepam (5 mg), perphenazine (4 mg), flurazepam $(7.5 \mathrm{mg})$. She was stable and had no stressful event. How- 
ever, at 2 months prior to the current hospital admission she had taken two tablets of terbinafine (Terbinafine HCL $250 \mathrm{mg}$ ) in 1 day due to onychomycosis of the toenails, after which she experienced extrapyramidal symptoms. Her symptoms did not disappear for 6 weeks, and so at 2 weeks prior to this most recent admission she had stopped taking terbinafine. Her symptoms persisted despite discontinuing the terbinafine, leading her family bringing her to my hospital. Her baseline scores on the Hamilton Depression Rating Scale, ${ }^{5}$ SimpsonAngus Scale ${ }^{6}$ and Barnes Akathisia Rating Scale ${ }^{7}$ were 23, 11, and 9, respectively. We discontinued her psychiatric medication, including perphenazine and added mirtazapine, clonazepam, and propranolol. Her symptoms improved rapidly, such that 1 week later the only symptom that persisted was insomnia. She was otherwise well. Her 1 week scores on the Hamilton Depression Rating Scale ${ }^{5}$, Simpson-Angus Scale ${ }^{6}$ and Barnes Akathisia Rating Scale ${ }^{7}$ were 7, 2, and 3, respectively.

\section{DISCUSSION}

I present here a patient receiving perphenazine with extrapyramidal symptoms during terbinafine therapy that persisted after the discontinuation of terbinafine therapy. The antifungal agent terbinafine is highly lipophilic and keratophilic, ${ }^{3}$ and is distributed extensively throughout the adipose tissue, dermis, epidermis, and nails in humans. ${ }^{8}$ Moreover, some studies have shown that terbinafine is eliminated in a triphasic pattern with a $t_{1 / 2 \gamma}$ of 17-32 days, consistent with a depot effect. ${ }^{9,10}$ Thus, terbinafine could be involved in both long lasting drug-drug interactions and interactions appearing weeks after terbinfine discontinuation. ${ }^{2}$ In addition, it was found that the clearance of perphenazine is significantly lower in CYP2D6-deficient individuals. ${ }^{11}$ It was also reported a case on a 37-year-old white woman with normal CYP2D6 metabolic capacity who was treated with amitriptyline when terbinafine was introduced. ${ }^{3}$ Shortly thereafter she experienced extreme adverse effects accompanied by a large increase in the serum concentrations of amitriptyline. Terbinafine therapy was discontinued, and the amitriptyline dose was reduced. Surprisingly, the serum concentrations of amitriptyline did not return to baseline until approximately 6 months later. ${ }^{3}$ This may cause serious adverse effects when terbinafine is combined with drugs that have a relatively narrow therapeutic index, e.g., antipsychotics (perphenazine, zuclopentixol, aripiprazole, risperidone), tricyclic antidepressants (amitriptyline, nortriptyline, imipramine, desipramine, clomipramine), and b-blockers (metoprolol, timolol). ${ }^{1,3,12-14}$

The patient described herein was stable before terbinafine therapy. There was thus a temporal relationship between extrapyramidal symptoms and terbinafine therapy. These symp- toms also disappeared 3 weeks after discontinuing the terbinafine therapy. In addition, the administration of propranolol and mirtazapine, which are known to be effective for the treatment of antipsychotic-induced akathisia, ${ }^{15}$ rapidly resolved her akathisia and anxiety. Thus, it is possible that a drug-drug interaction between terbinafine and perphenazine induced this patient's extrapyramidal symptoms, including the akathisia.

However, this case presents limitations. The concentration of terbinafine or perphenazine was not measured during terbinafine therapy or after the discontinuation of terbinafine therapy. In addition, the findings of a single case report cannot be generalized.

In conclusion, terbinafine therapy may be associated with the induction of extrapyramidal symptoms, including akathisia, which may persist even the discontinuation of terbinafine therapy. This case report emphasizes the importance of monitoring drug-drug interactions in patients undergoing terbinafine and perphenazine therapy.

\section{Acknowledgments}

This study was supported by a grant from National Research Foundation of Korea (NRF), funded by Ministry of Education and Science Technology (MEST) (2011-0010562).

\section{REFERENCES}

1. Venkatakrishnan K, von Moltke LL, Greenblatt DJ. Effects of the antifungal agents on oxidative drug metabolism: clinical relevance. Clin Pharmacokinet 2000;38:111-180.

2. Levine B, Jenkins A, Chute D, Smialek JE. Perphenazine distribution in a postmortem case. J Anal Toxicol 1999;23:127-129.

3. Castberg I, Helle J, Aamo TO. Prolonged pharmacokinetic drug interaction between terbinafine and amitriptyline. Ther Drug Monit 2005; 27:680-682.

4. Schatz F, Brautigam M, Dobrowolski E, Effendy I, Haberl H, Mensing $\mathrm{H}$, et al. Nail incorporation kinetics of terbinafine in onychomycosis patients. Clin Exp Dermatol 1995;20:377-383.

5. Hamilton M. A rating scale for depression. J Neurol Neurosurg Psychiatry 1960;23:56-62.

6. Simpson GM, Angus JW. A rating scale for extrapyramidal side effects. Acta Psychiatr Scand Suppl 1970;212:11-19.

7. Barnes TR. A rating scale for drug-induced akathisia. Br J Psychiatry 1989;154:672-676.

8. Faergemann J, Zehender H, Denouël J, Millerioux L. Levels of terbinafine in plasma, stratum corneum, dermis-epidermis (without stratum corneum), sebum, hair and nails during and after $250 \mathrm{mg}$ terbinafine orally once per day for four weeks. Acta Derm Venereol 1993;73:305309.

9. Zehender F, Cabiac MD, Denouël J, Faergemann J, Donatsch P, Kutz K, et al. Elimination kinetics of terbinafine from human plasma and tissues following multiple dose administration and comparison with 3 main metabolites. Drug Invest 1994;8:203-210.

10. Kovarik JM, Mueller EA, Zehender H, Denouël J, Caplain H, Millerioux L. Multiple-dose pharmacokinetics and distribution in tissue of terbinafine and metabolites. Antimicrob Agents Chemother 1995;39: 2738-2741.

11. Dahl-PuustinenM-L, Lidén A, Alm C, Nordin C, Bertilsson L. Disposition of perphenazine is related to polymorphic debrisoquin hydroxylation in human beings. Clin Pharmacol Ther 1989;46:78-81. 
12. Venkatakrishnan K, Obach RS. In vitro-in vivo extrapolation of CYP2D6 inactivation by paroxetine: prediction of nonstationary pharmacokinetics and drug interaction magnitude. Drug Metab Dispos 2005; 33:845-852.

13. Abdel-Rahman SM, Marcucci K, Boge T, Gotschall RR, Kearns GL, Leeder JS. Potent inhibition of cytochrome P-450 2D6-mediated dextromethorphan O-demethylation by terbinafine. Drug Metab Dispos 1999;27:770-775.
14. Molden E, Garcia BH, Braathen P, Eggen AE. Co-prescription of cytochrome $\mathrm{P} 450$ 2D6/3A4 inhibitor-substrate pairs in clinical practice. A retrospective analysis of data from Norwegian primary pharmacies. Eur J Clin Pharmacol 2005;61:119-125.

15. Poyurovsky M, Pashinian A, Weizman R, Fuchs C, Weizman A. Lowdose mirtazapine: a new option in the treatment of antipsychotic-induced akathisia. A randomized, double-blind, placebo- and propranololcontrolled trial. Biol Psychiatry 2006;59:1071-1077. 